\title{
Association between the MHC gene region and variation of serum IgE levels against specific mould allergens in the horse
}

\author{
Ino CURIK ${ }^{\mathrm{a}}$, Darrilyn Fraser ${ }^{\mathrm{b}}$, Claudia Eder ${ }^{\mathrm{c}}$, \\ Roland AchmanN ${ }^{\mathrm{d}}$, June Swinburne ${ }^{\mathrm{e}}$, Matthew Binns ${ }^{\mathrm{e}}$, \\ Reto CrAMERI ${ }^{\mathrm{f}}$, Gottfried BREM ${ }^{\mathrm{d}}$, \\ Johann SÖLKNER $^{\mathrm{g}}$, Eliane MARTI ${ }^{\mathrm{h} *}$ \\ ${ }^{a}$ Animal Science Department, Faculty of Agriculture, University of Zagreb, \\ Svetosimunska 25, 10000-Zagreb, Croatia \\ ${ }^{\mathrm{b}}$ Department of Veterinary Microbiology and Pathology, \\ Washington State University, Pullman, Washington 99164-7040, USA \\ ${ }^{c}$ Division of Immunogenetics, Institute of Animal Breeding, University of Berne, \\ Bremgartenstrasse 109 A, 3012-Berne, Switzerland \\ ${ }^{\mathrm{d}}$ Ludwig-Boltzmann-Institute for Cyto-, Immuno- and Molecular Genetic Research, \\ Veterinärplatz 1, 1210-Vienna, Austria \\ e Animal Health Trust, Newmarket, Suffolk CB8 7UU, UK \\ ${ }^{f}$ Swiss Institute of Allergy and Asthma Research (SIAF), Obere Strasse 22, \\ 7270-Davos, Switzerland \\ g Department of Livestock Science, University of Agricultural Sciences, \\ Gregor-Mendel-Strasse 33, 1180-Vienna, Austria \\ ${ }^{\mathrm{h}}$ Division of Clinical Immunology, Department of Clinical Veterinary Medicine, \\ University of Berne, Länggass-Strasse 124, 3012-Berne, Switzerland
}

(Accepted 26 February 2003)

\begin{abstract}
To investigate whether the equine major histocompatibility complex $(M H C)$ gene region influences the production of mould-specific immunoglobulin $\mathrm{E}$ antibodies (IgE), alleles of the equine leukocyte antigen (ELA-A) locus and three microsatellite markers (UM-011, $H T G-05$ and $H M S-42$ ) located on the same chromosome as the equine MHC were determined in 448 Lipizzan horses. Statistical analyses based on composite models, showed significant associations of the ELA-A and UM-011 loci with IgE titres against the recombinant Aspergillus fumigatus 7 antigen ( $r A s p f 7$ ). UM-011 was also significantly associated with IgE titres against the recombinant Aspergillus fumigatus 8 antigen ( $r A s p f 8$ ). In addition to the loci mentioned above, the MHC class II $D Q A$ and $D R A$ loci were determined in 76 Lipizzans from one stud. For IgE levels against $r A s p f 7$, the composite model showed the strongest association for $D Q A$
\end{abstract}

* Correspondence and reprints

E-mail: eliane.marti@itz.unibe.ch 
$(P<0.01)$ while for $r A s p f 8$ specific IgE levels, similarly to the results found with all 448 horses, the strongest association was found with $U M-011(P=0.01)$, which is closely linked with the MHC class II $D R B$ locus. These results suggest that the equine MHC gene region and possibly MHC class II loci, influence the specific IgE response in the horse. However, although the strongest associations were found with $D Q A$ and $U M-011$, this study did not distinguish if the observed effects were due to the MHC itself or to other tightly linked genes.

horse / major histocompatibility complex / immunoglobulin E / specific mould allergen

\section{INTRODUCTION}

Immunoglobulin $\mathrm{E}(\mathrm{IgE})$ is an antibody class which mediates immediate type hypersensitivity reactions and plays an important role in the pathogenesis of allergic diseases and in the defence against helminths. Allergic diseases are hypersensitivity reactions of certain individuals to substances called allergens, often present in the natural environment, which are usually harmless for the majority of the population. Production of specific $\operatorname{IgE}$ is initiated by the uptake and presentation of allergens by major histocompatibility complex (MHC) class II positive cells to allergen-specific helper $\mathrm{T}$ lymphocytes. These $\mathrm{T}$ lymphocytes instruct B lymphocytes to produce allergen-specific IgE antibodies. Allergen-specific IgE antibodies bind to the high affinity receptors present on mast cells and basophils and allergen-induced IgE cross-linking results in the release of various inflammatory mediators.

In human studies of family aggregation and segregation, it has been suggested that total serum IgE levels are under genetic control and are associated with, or linked to a number of genes [2], although the mechanism of inheritance has not been determined. In addition to genetic factors which influence a general predisposition for high IgE levels, MHC class II genes, and more precisely $H L A-D$ genes, are involved in the specific immune responsiveness to allergens [2]. Family studies have also demonstrated a genetic predisposition for IgE-mediated allergic diseases, such as asthma in humans [5].

In the horse, recurrent airway obstruction (RAO), which has some similarities with human asthma, is caused by hypersensitivity reactions to moulds and possibly also to other components present in hay and straw dusts [19]. IgE-mediated immune responses are probably involved in the pathogenesis of RAO: affected horses have higher IgE levels against mould extracts [12,21] and against certain recombinant (r) mould allergens [6] than healthy control horses. A genetic predisposition for RAO has been demonstrated with family studies [15], but an association between the equine leukocyte antigens (ELA) and RAO has not been found [15]. This lack of association is not surprising, since RAO is probably caused by immune reactions against a large number of different antigens and associations with the MHC are only found with specific IgE responses against single, well-defined allergenic molecules [2]. 
A recent study in Lipizzan horses has shown that allergen-specific serum $\operatorname{IgE}$ levels are influenced by environmental and genetic factors [7]. A preliminary investigation also suggested that some MHC class I alleles, also called equine leukocyte antigens (ELA), are associated with the IgE response against mould allergens [7].

In order to further investigate whether the MHC gene region influences the allergen-specific IgE response in the horse, three microsatellite markers located on the same chromosome (ECA-20) as the equine MHC [23] were typed on 448 Lipizzan horses from six national studs. One of these markers, UM-011, is closely linked with the MHC class II DRB locus [23].

Furthermore, alleles of the MHC class II $D Q A$ and $D R A$ loci were determined in 76 Lipizzan horses from one stud. IgE levels against two mould extracts and against three recombinant mould allergens had been determined previously in the sera of these horses [7].

\section{MATERIALS AND METHODS}

\subsection{Horses}

Four-hundred and forty-eight Lipizzan horses, brood mares and stallions, belonging to six studs from six different countries were included in the study: Austria: Piber stud (153 horses); Croatia: Djakovo stud (56 horses); Italy: Monterotondo stud (61 horses); Hungary: Szilvásvárad stud (76 horses); Slovakia: Topol'cianky stud (42 horses); and Slovenia: Lipizza stud (59 horses). All tested horses belonged to two generations (102 parent-offspring pairs) and were over three years old. A detailed description of the population structure is reported in Zechner et al. [25]. Within each stud, the horses were exposed to similar environmental conditions and fed the same hay. All mares were kept loose in group housing stables during the winter and were at pasture and in stables during the summer. The stallions were held in individual loose boxes in stables separate from the mares, but were fed the same hay. A clinical examination of the respiratory tract permitting to identify horses suffering from RAO could unfortunately not be carried out because the brood mares of a number of studs were not used to handling, rending a thorough examination of the respiratory tract impossible without sedation. Although we did not see horses severely affected with RAO, coughing animals were present, but we could not exclude infections of the respiratory tracts and thus did not include the diagnosis as a variable in the statistical analysis. We can expect from previous studies [19] that at least $10 \%$ and probably more horses were affected with RAO.

\subsection{Blood samples}

Blood was taken from the jugular vein from each horse with sodium heparin containing vacutainers for the serological MHC typing, with EDTA containing 
vacutainers for DNA isolation and with vacutainers (Vacuette ${ }^{\circledR}$ Greiner, Huber u. Co., Reinach, Switzerland) containing a serum clot activator for preparation of the serum. Serum was frozen in aliquots within $24 \mathrm{~h}$ after blood sampling and then stored at $-70^{\circ} \mathrm{C}$ until assayed.

\subsection{Serological MHC class I typing}

Alloantisera detecting 17 internationally recognised (A1, A2, A3, A4, A5, A6, A7, A8, A9, A10, W11, A14, A15, A16, A17, A18, A19 and A20) and six locally defined (Be22, Be25, Be27, Be28, Be30 and Be108) ELA-A locus specificities were tested in a classical two-step microcytotoxicity test in Terasaki plates according to Lazary et al. $[13,14]$.

\subsection{Isolation of DNA}

DNA was isolated from whole blood using a Nucleon DNA Extraction Kit (Amersham Biosciences UK Limited, Buckinghamshire, England) following the manufacturer's instructions.

\subsection{Determination of MHC class II alleles}

MHC class II $D R A$ and $D Q A$ locus typing was performed by the polymerase chain reaction-single strand conformation polymorphism (PCR-SSCP) analysis of exon 2 according to previously published protocols $[1,10]$ in the 76 horses from the Szilvásvárad stud. Typing for DRB [9] was performed but the data was not included in the analysis because of unreliable results.

\subsection{Genotyping of microsatellite markers}

Microsatellite markers $U M-011, H T G-05$ and $H M S$-42 mapping to equine chromosome 20 (ECA-20; Swinburne et al. [23]) were genotyped in the 448 horses. While the $U M-011$ locus is placed within MHC class II (no recombination between $U M-011$ and DRB [23]), the other two microsatellites (HTG-05 and $H M S-42)$, here used as flanking controls, are located proximal and distal on ECA-20. The three primer pairs (http://www.aht.org.uk/genetics/table2.html and http://locus.jouy.inrafr/cgi-bin/horsemap/) were pooled into a PCRmultiplex performed as described in Swinburne et al. [23].

\subsection{Determination of allergen-specific IgE levels in the horse}

Serum IgE levels against Alternaria alternata (Alt a) and Aspergillus fumigatus (Aspf) extracts, and against recombinant Alternaria alternata 1 (rAlt a 1), recombinant Aspergillus fumigatus 7 and 8 ( $r A s p f 7$ and $r A s p f 8$ ) were determined by enzyme linked immunoassay (ELISA) as described previously $[6,7]$. 


\subsection{Allele frequencies and associations between $E C A-20$ loci}

Allele frequencies were derived from the genotypic frequencies. Assumptions of the Hardy-Weinberg equilibrium (HWE) were tested for each locus within studs by the Markov-chain Monte Carlo algorithm implemented in the Genepop program version 3.2a [18], which generates exact tests for multiple alleles [11]. Bonferoni corrections (six comparisons per locus) were used on the HWE tests to reduce the Type I error rate. Associations between ECA-20 loci were presented as correlation coefficients, i.e. unbiased linkage disequilibrium coefficients normalised by allele frequencies, between combinations of loci or alleles included in the study and were statistically tested according to Weir [24]. The analysis was performed by the stud as well as on the pooled sample with all horses. Both the calculation of the correlation coefficients and their significance were performed by GENETIX 4.02 [3].

\subsection{Effects of $E C A-20$ loci on IgE level}

Variance analyses of the IgE levels were performed by the $\mathrm{SAS}^{\circledR}$ statistical package [20]. General linear models (PROC GLM) were used for normally distributed variables, serum IgE levels against the mould extracts Alternaria alternata (Alt a) and Aspergillus fumigatus (Asp $f$ ). The recombinant mould allergens rAlt a 1, rAsp $f 7$ and $r A s p f 8$ were treated as binary variables (detectable or undetectable IgE level) and were analysed with generalised linear models (PROC GENMOD) assuming a binomial error distribution [22]. In all models, response variables were analysed separately (univariate approach). The following procedure was applied in all cases. First, we fitted a model that included the effects of the stud (fixed), sex (fixed), age at measurement (covariable) and all possible two-way interactions between these effects. Then, starting from the higher order terms to lower order terms, the predictor variables having the highest $P$ value were omitted from the model if they were nonsignificant. Later, full-composed genetic models including all loci effects were simultaneously fitted on the minimal non-genetic models. Repeating the same modelling strategy as before (reduction of non-significant effects with the highest $P$ value), we built the resultant genetic model. A description of the models used in the analysis is shown in Table I.

The effects of the ECA-2O loci on the IgE response against the mould extracts as well as against the recombinant mould allergens were tested by gene substitution models [8]. Dependences that occured in a gene substitution model were removed [16]. All rare alleles with a frequency of less than $4 \%$ were pooled (see Tabs. IV and VI). This procedure enabled us to reduce the chance of making a type I error. The same modelling strategy (for description of the models used see Tab. I) was repeated for the sample with the 76 horses from the Szilvásvárad stud with addition of two MHC class II loci (DQA and $D R A)$. 
Table I. Description of the models used in the analysis with respect to the non-genetic effects, ECA-20 loci involved, statistical analysis (logistic and linear regression), response variables (Alt a, Asp f, rAlt a 1, rAsp $f 7$ and $r A s p f 8$ ) and dataset used (all studs and Szilvásvárad stud only).

\begin{tabular}{|c|c|c|c|c|c|}
\hline \multirow[t]{2}{*}{ Effects } & \multicolumn{2}{|c|}{ Linear regression } & \multicolumn{3}{|c|}{ Logistic regression } \\
\hline & Alternaria $a$. & Aspergillus $f$. & rAlt a 1 & $r A s p f 7$ & $r A s p f 8$ \\
\hline \multicolumn{6}{|c|}{ All studs $(\mathrm{N}=448)$} \\
\hline Stud & $* * *$ & $* * *$ & $* * *$ & $* * *$ & $* * *$ \\
\hline Age & $*$ & $* *$ & ns & ns & ns \\
\hline Sex & ns & ns & ns & ns & ns \\
\hline Stud $\times$ Age & *** & $* * *$ & ns & $*$ & ns \\
\hline Stud $\times$ Sex & $* * *$ & $* * *$ & ns & ns & ns \\
\hline$E C A-20$ loci & \multicolumn{5}{|c|}{ ELA-A, HMS-42, HTG-05 and UM-011 } \\
\hline
\end{tabular}

Szilvásvárad $(\mathrm{N}=76)$

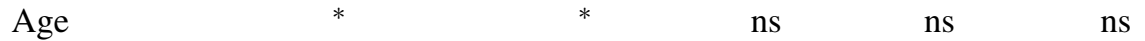

ECA-20 loci $\quad$ DQA, DRA, ELA-A,HMS-42, HTG-05 and UM-011

Significance of non-genetic effects: $\mathrm{ns}=$ not significant, ${ }^{*} P \leq 0.05,{ }^{* *} P \leq 0.01$ and ${ }^{* * *} P \leq 0.001 . \mathrm{N}=$ number of horses.

Since the number of horses was much lower (76), in these models, all rare alleles with a frequency of less than $5 \%$ were pooled (see Tabs. V and VII).

Overdominance effects were tested according to the symmetrical overdominance model [17]. Under symmetrical overdominance model values of homozygotes were compared against heterozygotes. However, there was no single significant association observed and, thus, the results related to this model are not further presented.

\section{RESULTS}

\subsection{Population genetics of the ECA-20 loci}

Fifteen different equine leukocyte antigen A $(E L A-A)$ alleles were detected on the typed Lipizzan horses (Tab. IV). At the $D Q A$ locus, four alleles out of eight accounted for $94 \%$ of all alleles (see Tab. V). At the DRA locus, three alleles were identified; DRA*0101 had the highest allele frequency $(53.3 \%)$, followed by DRA*0201 (33.8\%) and DRA*0301 (11.9\%). Amongst the three microsatellite loci (HTG-05, UM-011 and HMS-42), the largest number of alleles (10) was observed at the UM-011 locus. Four alleles $(161,163,165$ and 177 ) with frequencies ranging from $14.1 \%$ to $32.8 \%$ accounted for $89.8 \%$ of all alleles, (Tab. VI). Only five and four alleles were found at the HTG-05 and $H M S-042$ loci, respectively. At both loci, two alleles were predominant. The HTG-05-79 and HTG-05-81 alleles appeared with frequencies of 35.9\% 
Table II. Correlation coefficients (normalised disequilibrium coefficients) between loci on equine chromosome 20 (ECA-20).

\begin{tabular}{llllll}
\hline Locus & $H M S-42$ & $H T G-05$ & $U M-011$ & $E L A-A$ & $D Q A$ \\
\hline All studs (N=448) & & & & & \\
ELA-A & 0.052 & $0.059^{* * *}$ & $0.094^{* * *}$ & & \\
$H M S-42$ & & 0.038 & 0.046 & & \\
$H T G-05$ & & & 0.045 & & \\
\hline Szilvásvárad (N = 76) & & & & & \\
$D R A$ & 0.080 & 0.171 & $0.337^{* * *}$ & $0.213^{* * *}$ & $0.380^{* * *}$ \\
$H M S-42$ & & 0.114 & $0.140^{*}$ & 0.118 & 0.081 \\
$H T G-05$ & & & 0.100 & 0.089 & $0.116^{*}$ \\
$U M-011$ & & & & $0.220^{* * *}$ & $0.299^{* * *}$ \\
$E L A-A$ & & & & $0.241^{* * *}$ \\
\hline${ }^{*} P<0.05,{ }^{* *} P<0.01$ and $^{* * *} P<0.001 . \mathrm{N}=$ number of horses. &
\end{tabular}

and $53.1 \%$, respectively. A similar situation was observed at the HMS-042 locus; the frequencies of the HMS-042-112 and HMS-042-126 alleles were $40.2 \%$ and $47.6 \%$, respectively. All loci at all studs were in agreement with Hardy-Weinberg equilibrium.

ELA-A and $U M-011$ as well as ELA-A and HTG-05 were found to be in significant linkage disequilibrium (Tab. II). Analysis of pairwise allele associations showed significant correlations between certain alleles. For example, between alleles ELA-A1 and UM-011-163, ELA-A8 and UM-011-173, ELA-A14 and UM-011-163, and ELA-A16 and UM-011-177 significant $(P<0.0001)$ correlation coefficients of $0.451,0.865,0.471$ and 0.372 were observed, respectively. For the small dataset (76 horses), the highest significant correlation coefficients were found between the two MHC class II loci, $D Q A$ and $D R A$, as well as between $D R A$ and $U M-011$, and $D Q A$ and $U M-011$. Furthermore, highly significant correlation coefficients were observed between $E L A-A$ and $D Q A$, ELA-A and UM-011, and ELA-A and DRA (Tab. II).

\subsection{Association between ECA-20 loci and IgE levels against the mould extracts}

No significant overall effect (gene substitution model) of any of the loci considered was found for the IgE serum against the mould extracts Alt $a$ and Aspf.

\subsection{Association between $E C A-20$ loci and IgE levels against the recombinant mould allergens}

Although, for the model with 76 horses (Szilvásvárad stud), the effect of microsatellite $U M-011$ nearly reached the level of significance $(P=0.054)$, 
Table III. Associations between the effects of different loci on equine chromosome 20 (ECA-20) and serum IgE (detectable or undetectable) against the recombinant mould allergens: rAspergillus fumigatus 7 and 8 ( $r A s p f 7$ and $r A s p f 8)$.

\begin{tabular}{|c|c|c|c|c|c|}
\hline \multirow[b]{2}{*}{ Loci } & \multirow[b]{2}{*}{ df } & \multicolumn{2}{|c|}{$r A s p f 7$} & \multicolumn{2}{|c|}{$r A s p f 8$} \\
\hline & & $\Delta$ deviance & $P$-value & $\Delta$ deviance & $P$-value \\
\hline \multicolumn{6}{|c|}{ All studs $(\mathrm{N}=448)$} \\
\hline$H M S-42$ & 2 & 2.8479 & $0.24076(2)$ & 0.5630 & $0.75464(1)$ \\
\hline HTG-05 & 3 & 0.4902 & $0.92104(1)$ & 7.1736 & $0.06657(2)$ \\
\hline$U M-011$ & 4 & 11.5693 & 0.02086 & 17.3382 & 0.00164 \\
\hline$E L A-A$ & 10 & 20.0937 & 0.02838 & 17.3630 & $0.06721(3)$ \\
\hline \multicolumn{6}{|c|}{ Szilvásvárad $(\mathrm{N}=76)$} \\
\hline$H M S-42$ & 2 & 0.9267 & $0.62919(3)$ & 2.5502 & $0.27940(2)$ \\
\hline$H T G-05$ & 2 & 0.0471 & $0.97670(1)$ & 6.9844 & 0.03043 \\
\hline$U M-011$ & 5 & 7.2052 & $0.20582(2)$ & 14.3739 & 0.01340 \\
\hline$E L A-A$ & 5 & 10.1250 & $0.07177(4)$ & 2.9731 & $0.70413(1)$ \\
\hline$D Q A$ & 4 & 13.6297 & 0.00858 & 4.7143 & $0.31789(4)$ \\
\hline$D R A$ & 2 & 4.6211 & $0.09921(5)$ & 3.2354 & $0.19835(3)$ \\
\hline
\end{tabular}

Status of the locus (in parentheses) relates to the order of deletion of certain loci from the model: $(1)=$ first deleted, $\ldots,(5)=$ fifth deleted. $\mathrm{N}=$ number of horses.

no significant overall loci effects on serum IgE levels against $r$ Alt a 1 were obtained (data not shown).

On the contrary, significant overall loci effects (Tab. III) were obtained when the analysis was performed for serum IgE levels against $r A s p f 7$ or $r A s p f 8$.

When the analysis was performed with the 448 horses, UM-011 and ELA-A were significantly associated $(P<0.05)$ with the presence or absence of $\operatorname{IgE}$ serum against $r A s p f 7$.

Significant positive effects were detected for alleles UM-011-177 and ELAA1, while a significant negative effect was detected for the ELA-A8 allele (Tab. IV).

In the model including only the 76 horses from Szilvasvarad stud, a significant association $(P<0.01)$ was obtained between the overall effect of $D Q A$ and the IgE response to $r A s p f 7$. As shown in Table $\mathrm{V}$, a significant positive effect $(P<0.01)$ was observed for the DQA*0301 allele.

A significant association was also obtained between microsatellite UM-011 and serum IgE against $r A s p f 8$ (Tab. III) when the horses from all studs were analysed. Again, as was the case for the $\operatorname{IgE}$ response to $r A s p f 7$, the UM-011-177 allele was significantly positively associated with IgE against rAsp $f 8$ (Tab. VI). In addition, a significant negative effect was observed (Tab. VI) for the UM-011-161 allele as well as for the pooled rare alleles. 
Table IV. Gene substitution effect $(\alpha)$ for $E L A-A$ and microsatellite $U M-011$ loci on the IgE response to recombinant Aspergillus fumigatus [ $r A s p f 7]$ in the model with 448 horses.

\begin{tabular}{lcccc}
\hline Locus & Allele & Frequency & \multicolumn{2}{c}{$\alpha(\mathrm{SE})$} \\
\hline ELA-A & A1 & 14.6 & $0.71^{*}(0.31)$ \\
A2 & 7.4 & -0.50 & $(0.44)$ \\
A3 & 4.5 & 0.28 & $(0.49)$ \\
A4 & 5.2 & 0.37 & $(0.46)$ \\
A7 & 6.0 & 0.54 & $(0.39)$ \\
A8 & 4.5 & $-1.63^{* *}(0.61)$ \\
A14 & 5.9 & 0.78 & $(0.48)$ \\
A16 & 12.2 & -0.39 & $(0.35)$ \\
A19 & 22.6 & -0.58 & $(0.30)$ \\
Be27 & 11.2 & 0.31 & $(0.36)$ \\
& Pooled rare alleles & 5.7 & 0.10 & $(0.42)$ \\
\hline 161 & 26.1 & -0.13 & $(0.23)$ \\
163 & 17.8 & -0.51 & $(0.28)$ \\
& 165 & 32.8 & -0.11 & $(0.20)$ \\
& 177 & 14.1 & $0.81^{* *}(0.26)$ \\
& Pooled rare alleles & 10.2 & -0.05 & $(0.37)$ \\
\hline
\end{tabular}

Gene substitution effects $(\alpha)$ are derived from a generalised linear model assuming a binomial error distribution. ${ }^{*} P<0.05,{ }^{* *} P<0.01$. ${ }^{\#}$ For the microsatellite marker $U M-011$ the alleles relate to the size in bp. Pooled rare alleles: ELA-A (A10, A11, A17, A20 and Be30) and $U M-011$ (159, 164, 167, 171, 172 and 173).

Table V. Gene substitution effect $(\alpha)$ for the $D Q A$ locus on the IgE response to recombinant Aspergillus fumigatus [rAsp $f$ 7] in the model with 76 horses.

\begin{tabular}{lcrc}
\hline Allele & Frequency & $\alpha(\mathrm{SE})$ \\
\hline $\mathrm{DQA}^{* 0301}$ & 12.7 & $1.33^{* *}(0.47)$ \\
$\mathrm{DQA}^{*} 0601$ & 34.7 & 0.04 & $(0.28)$ \\
$\mathrm{DQA}^{*} 0801$ & 25.3 & -0.44 & $(0.32)$ \\
DQA*1401 & 19.3 & -0.77 & $(0.43)$ \\
Pooled rare alleles & 6.0 & -0.16 & $(0.54)$ \\
\hline
\end{tabular}

Gene substitution effects $(\alpha)$ are derived from the generalised linear model assuming a binomial error distribution. ${ }^{*} P<0.05,{ }^{* *} P<0.01$. Pooled rare alleles: $D Q A$ $(* 0201, * 0501, * 0901$ and $* 1201)$. 
Table VI. Gene substitution effect $(\alpha)$ for the microsatellite $U M$-011 locus on the IgE response to recombinant Aspergillus fumigatus $8[\mathrm{rAsp} f 8]$ in the model with 448 horses.

\begin{tabular}{lcc}
\hline Allele (size in bp) & Frequency & $\alpha(\mathrm{SE})$ \\
\hline 161 & 26.1 & $-0.51^{*}(0.23)$ \\
163 & 17.8 & $0.24 \quad(0.23)$ \\
165 & 32.8 & $0.24 \quad(0.18)$ \\
177 & 14.1 & $0.75^{* *}(0.25)$ \\
Pooled rare alleles & 10.2 & $-0.72^{*}(0.36)$ \\
\hline
\end{tabular}

Gene substitution effects $(\alpha)$ are derived from the generalised linear model assuming a binomial error distribution. ${ }^{*} P<0.05,{ }^{* *} P<0.01$. Pooled rare alleles: $U M-011$ $(159,164,167,171,172$ and 173).

Table VII. Gene substitution effect $(\alpha)$ for microsatellite loci $H T G$-05 and UM-011 on the IgE response to recombinant Aspergillus fumigatus 8 [ $\mathrm{KAsp} f 8]$ in the model with 76 horses.

\begin{tabular}{|c|c|c|c|}
\hline Locus & Allele (size in bp) & Frequency & $\alpha(\mathrm{SE})$ \\
\hline \multirow[t]{3}{*}{ HTG-05 } & 79 & 46.7 & $8.56^{*}(0.50)$ \\
\hline & 81 & 46.1 & $8.46^{*}(0.00)$ \\
\hline & 85 & 6.6 & $-17.02^{* *}(0.50)$ \\
\hline \multirow[t]{6}{*}{$U M-011$} & 161 & 23.7 & $-0.54 \quad(0.58)$ \\
\hline & 163 & 10.5 & $-0.17^{*}(0.65)$ \\
\hline & 165 & 34.2 & $0.84 \quad(0.42)$ \\
\hline & 173 & 17.1 & $-1.46^{*}(0.70)$ \\
\hline & 177 & 7.9 & $0.22 \quad(0.61)$ \\
\hline & Pooled rare alleles & 6.6 & $1.11 \quad(0.72)$ \\
\hline
\end{tabular}

Gene substitution effects $(\alpha)$ are derived from the generalised linear model assuming a binomial error distribution. ${ }^{*} P<0.05,{ }^{* *} P<0.01$. Pooled rare alleles: $U M-011$ (167, 171 and 172).

Microsatellite $U M-011$ in combination with the flanking marker $H T G-05$, was also significantly associated with the IgE response to $r A s p f 8$ when the analysis was performed within the Szilvásvárad stud (Tab. VII). While all three alleles of the HTG-05 locus exerted significant effects, only two alleles of microsatellite locus UM-011 (UM-011-163 and UM-011-173) were significantly associated with the IgE response against $r A s p f 8$ (Tab. VII).

No significant effects of the MHC class II loci DQA and DRA could be demonstrated for IgE levels against this r-allergen. 


\section{DISCUSSION}

A preliminary investigation indicated a possible association between the MHC gene region and allergen-specific IgE levels in the horse [7]. The present study confirmed these findings and showed that MHC class I and class II genes and markers linked to the equine MHC, are associated with IgE levels against recombinant Aspergillus fumigatus allergens but not with IgE levels against the mould extracts Aspergillus fumigatus and Alternaria alternata. It is not surprising that no significant associations were found with the mould extracts, since these extracts contain a high number of different proteins and glycoproteins and horses probably have IgE against many different antigens present in these extracts. Associations between specific IgE levels and the MHC can only be expected with single and pure allergens [2]. The data available made it possible to account for different sources of variation (stud, sex and age and their two-way interactions) that are known to affect serum IgE levels [7]. The determination of microsatellite markers linked to the equine MHC also allowed a more sophisticated statistical analysis than in the preliminary study of Eder et al. [7]. The present investigation included the MHC class I ELA-A locus and a microsatellite marker, $U M-011$, that is closely linked to the MHC class II $D R B$ locus, as well as two flanking microsatellite markers, $H T G-05$ and $H M S-42$. The composite models with 448 Lipizzans, fitting alleles from all four loci simultaneously, showed significant associations of the ELA-A and UM-011 loci with IgE titres against $r A s p f 7$, and of $U M-011$ solely with IgE against $r A s p f 8$. Based on the theoretical function of the MHC, i.e. presentation of endogenous peptides by class I and of exogenous peptides by class II gene products, we would assume an effect of MHC class II alleles on the IgE response against single allergens. To test this hypothesis, the alleles of the MHC class II loci, $D Q A$ and $D R A$, were determined in the 76 Lipizzans from the Szilvásvárad stud. This stud was selected particularly because it contained the highest percentage of horses with detectable $\operatorname{IgE}$ against $r A s p f 7$ and $r A s p f 8$ and a preliminary investigation [7] had indicated a negative association between an ELA-A allele and IgE levels against $r A s p f 7$ and $r A s p f 8$. The statistical analysis within the Szilvásvárad stud demonstrated the strongest association between $r A s p f 7$ specific IgE levels and the DQA locus, indicating that genes within the MHC class II rather than genes within the MHC class I region might be true candidates for the variability of serum IgE levels against $r A s p f 7$. The $U M-011$ locus, together with flanking marker $H T G-05$, showed a significant association with serum IgE levels against $r A s p f 8$. The significant association at locus HTG-05 is surprising but is likely caused by the strong effect of the rare allele, HTG05-85. With the exception of the association with $H T G-05$, which needs to be interpreted with caution, significant associations were only found with the MHC class II $D Q A$ loci and with the $D R B$-linked marker $U M$-011 further suggesting that the MHC class II loci may influence the specific IgE response in the horse. 
The readers should be aware that we did not account for the background genes and, thus, while estimating loci effects, possible bias might have arisen due to a non-random distribution of the polygenic components.

In conclusion, these results indicate that the MHC gene region, possibly MHC class II genes, influence the specific IgE response in the horse and that more than one class II locus might affect the specific allergen response, as has been suggested in humans [4]. A better characterisation of the equine MHC and a finer genetic map of ECA-20, as well as the identification of the relevant epitopes on the recombinant allergens will allow us to understand the mechanisms underlying the uncovered association between the equine MHC gene region and specific IgE levels. Further studies are also needed to investigate whether these findings are significant to the genetic resistance or susceptibility to recurrent airway obstruction in the horse.

\section{ACKNOWLEDGEMENTS}

We are grateful to the Directors of the studs for use of their horses and for the support of their staff when taking the blood samples. This work was supported by the Swiss Federal Office for Education and Science grant No. 96.0346, by the Swiss National Science Foundation grant No. 31-63449.00, by the Hans-Sigrist Foundation of the University of Berne and by the EU project INCO-COOPERNICUS No. IC15-CT96-0904. Work at SIAF was supported by the Swiss National Science Foundation grant No. 31-63381.00. Ino Curik is grateful to the "Österreicher Akademischer Austauschdienst" for financial support, as his contribution has been done while he was a visitor to the Department of Livestock Science, University of Agricultural Sciences Vienna, Austria. The Horserace Betting Levy Board and the Childwick Trust supported June Swinburne and Matthew Binns.

\section{REFERENCES}

[1] Albright-Fraser D.G., Reid R., Gerber V., Bailey E., Polymorphism of DRA among equids, Immunogenetics 43 (1996) 315-317, erratum Immunogenetics 44 (1996) 487.

[2] Barnes K.C., Marsh D.G., The genetics and complexity of allergy and asthma, Immunol. Today 19 (1998) 325-334.

[3] Belkhir K., Borsa P., Chikhi L., Raufaste N., Bonhomme F., GENETIX 4.02, logiciel sous Windows TM pour la génétique des populations, Laboratoire Génome, Populations, Interactions, Cnrs UMR 5000, Université Montpellier II, Montpellier, 2001.

[4] Collins A., Ennis S., Tapper W., Morton N.E., Mapping oligogenes for atopy and asthma by meta-analysis, Genet. Mol. Biol. 23 (2000) 1-10.

[5] Cookson W.O.C.M., Genetics and asthma, Res. Immunol. 149 (1998) 181-187. 
[6] Eder C., Crameri R., Mayer C., Eicher R., Straub R., Gerber H., Lazary S., Marti E., Allergen-specific IgE levels against crude mould and storage mite extracts and recombinant mould allergens in sera from horses affected with chronic bronchitis, Vet. Immunol. Immunopathol. 73 (2000) 241-253.

[7] Eder C., Curik I., Brem G., Crameri R., Bodo I., Habe F., Lazary S., Sölkner J., Marti E., Influence of environmental and genetic factors on allergen-specific immunoglobulin-E levels in sera from Lipizzan horses, Equine Vet. J. 33 (2001) 714-720.

[8] Falconer D.S., Mackay F.C.T., Introduction to Quantitative Genetics, Longman Group, London, 1996.

[9] Fraser D.G., Bailey E., Demonstration of three $D R B$ loci in a domestic horse family, Immunogenetics 44 (1996) 441-445.

[10] Fraser D.G., Bailey E., Polymorphism and multiple loci for the horse $D Q A$ gene, Immunogenetics 47 (1998) 487-490.

[11] Guo S.W., Thompson E.A., Performing the exact test of Hardy-Weinberg proportion for multiple alleles, Biometrics 48 (1992) 361-372.

[12] Halliwell R.E.W., McGorum P.C., Irving P., Dixon P.M., Local and systemic antibody production in horses affected with chronic pulmonary disease, Vet. Immunol. Immunopathol. 38 (1993) 201-215.

[13] Lazary S., De Weck A.L., Bullen S., Straub R., Gerber H., Equine leucocyte antigen system. I. Serological studies, Transplantation 30 (1980) 203-209.

[14] Lazary S., Antczak D.F., Bailey E., Bell T.K., Bernoco D., Byrns G., Mc Clure J.J., Joint Report of the Fifth International Workshop on Lymphocyte Alloantigens of the Horse, Baton Rouge, Louisiana, 31 October-1 November 1987, Anim. Genet. 19 (1988) 447-456.

[15] Marti E., Gerber H., Essich G., Ouhlela J., Lazary S., On the genetic basis of equine allergic diseases. I. Chronic hypersensitivity bronchitis, Equine Vet. J. 23 (1991) 457-460.

[16] Østergård H., Kristensen B., Andersen S., Investigations in farm animals of associations between the MHC system and disease resistance and fertility, Livest. Prod. Sci. 22 (1989) 49-67.

[17] Paterson S., Wilson K., Pemberton J.M., Major histocompatibility complex variation associated with juvenile survival and parasite resistance in a large unmanaged ungulate population (Ovis aries L.), Proc. Natl. Acad. Sci. USA 95 (1998) 3714-3719.

[18] Raymond M., Rousset F., Genepop (version 1.2) - population genetics software for exact tests and ecumenicism, J. Hered. 86 (1995) 248-249.

[19] Robinson N.E., Derksen F.J., Olszeweski M.A., Buechner-Maxwell V.A., The pathogenesis of chronic obstructive pulmonary disease of horses, Br. Vet. J. 152 (1996) 283-306.

[20] SAS $^{\circledR}$ Institute Inc. SAS/STAT Software: Changes and Enhancements through Release 6.11, Cary, NC, 1996.

[21] Schmallenbach K.H., Rahman I., Sasse H.H.L., Dixon P.M., Halliwell R.E.W., McGorum B.B., Crameri R., Miller H.R.P., Studies on pulmonary and systemic Aspergillus fumigatus specific $\mathrm{IgE}$ and $\mathrm{IgG}$ antibodies in horses affected with chronic obstructive pulmonary disease (COPD), Vet. Immunol. Immunopathol. 66 (1998) 245-256. 
[22] Stokes M.E., Davis C.S., Koch G.C., Categorical data analysis using the SAS ${ }^{\circledR}$ system, SAS ${ }^{\circledR}$ Institute, Cary, NC, 1995.

[23] Swinburne J., Gerstenberg C., Breen M., Aldridge V., Lockhart L., Marti E., Antczak D., Eggleston-Stot M., Bailey E., Mickelson J., Røend K., Lindgren G., von Haeringen W., Guérin G., Bjarnason J., Allen T., Binns M., First comprehensive low-density horse linkage map based on two 3-generation, full-sibling, cross-bred horse reference families, Genomics 66 (2000) 123-134.

[24] Weir B.S., Inferences about linkage disequilibrium, Biometrics 35 (1979) 235-254.

[25] Zechner P., Sölkner J., Druml T., Baumung R., Achmann R., Bodo I., Marti E., Habe F., Brem G., Analysis of diversity and population structure in the Lipizzan horse breed based on pedigrees information, Livest. Sci. Prod. 77 (2002) 137-146.

To access this journal online: www.edpsciences.org 